\title{
Allogeneic Chimeric Antigen Receptor Therapy in Lymphoma
}

\section{Arushi Khurana, MBBS $\odot$ Yi Lin, MD, PhD ${ }^{*}$}

\author{
Address \\ "Division of Hematology, Mayo Clinic, 200 First Street, SW, Rochester, MN, 55905, \\ USA \\ Email: Lin.Yi@mayo.edu
}

Published online: 25 February 2022

(C) The Author(s) 2022

This article is part of the Topical Collection on Lymphoma

Keywords CAR-T · Allogeneic CAR-T • Non-Hodgkin lymphoma

\section{Opinion statement}

The therapeutic armamentarium has significantly expanded since the approval of various CD19-targeting chimeric antigen receptor T cell (CAR-T) therapies in non-Hodgkin lymphoma (NHL). These CAR-Ts are patient-specific and require a complex, resource, and time-consuming process. While this appears promising, autologous CAR-Ts are limited due to the lack of accessibility, manufacturing delays, and variable product quality. To overcome these, allogeneic (allo) CARs from healthy donors appear appealing. These can be immediately available as "off the shelf" ready-to-use products of standardized and superior quality exempt from the effects of an immunosuppressive tumor microenvironment and prior treatments, and potentially with lower healthcare utilization using industrialized scale production. Allogeneic CARs, however, are not devoid of complications and require genomic editing, especially with $\alpha \beta T$ cells to avoid graft versus host disease (GvHD) and allo-rejection by the recipient's immune system. Tools for genomic editing such as TALEN and CRISPR provide promise to develop truly "off the shelf" universal CARs and further advance the field of cellular immunotherapy. Several allogeneic CARs are currently in early phase clinical trials, and preliminary data is encouraging. Longer followup is required to truly assess the feasibility and safety of these techniques in the patients. This review focuses on the strategies for developing allogeneic CARs along with cell sources and clinical experience thus far in lymphoma. 


\section{Introduction}

Chimeric antigen receptor $\mathrm{T}$ cell (CAR-T) therapy has been transformative for the treatment of relapsed/ refractory (R/R) large B cell lymphoma (LBCL) patients who have relapsed post 2 lines of therapy or autologous stem cell transplant (ASCT). These patients were otherwise subject to dismal outcomes [1]. Since 2017, three different CAR-T products have been approved by the US Food and Drug Administration (FDA) for the treatment of R/R LBCL after two or more prior lines of therapy [2•, $3 \bullet, 4 \bullet$ ]. All three boast a relatively similar durable response rate of around $40 \%$ at 2 years in the registration trials and real-world settings $[5-8,9]$. Two additional FDA approvals for lymphoma in the last year have been for mantle cell lymphoma (brexucabtagene autoleucel) and follicular lymphoma (axicabtagene ciloleucel) [10, 11•]. All the commercially approved CAR-T therapies are directed against CD19, a protein that is frequently expressed in B cell lymphomas, and in a relatively higher proportion than some other targets such as CD22 or CD20 [12]. The commercial production of autologous CAR-T cells requires the collection of $\mathrm{T}$ cells from the patient (leukapheresis), followed by activation and transduction with the CAR constructs using viral vectors, and eventually expansion before reinfusion into the patient after a short course of lymphodepleting chemotherapy [12]. This bespoke manufacturing process can take up to 2-5 weeks, a delay that may be particularly problematic for those with aggressive and rapidly proliferative lymphomas. Up to $30 \%$ of patients could not receive tisagenlecleucel CAR-T therapy in the JULIET trial, most of whom died due to progressive disease as the median time from leukapheresis to infusion was 54 days [13]. Additionally, while waiting for the cells to be manufactured, some patients require additional bridging therapy to maintain disease control which is associated with poor outcomes $[6 \bullet, 9,14]$. Delay in CAR-T therapy and increase in the lines of therapy before CAR-T have both been shown to worsen outcomes along with an increase in tumor burden, markers of inflammation, and doubling time of CAR-T cells $[15,16,17]$. Autologous CAR-Ts are expensive and laborious to manufacture due to patient specificity, vary in quality based on patient's disease factors and prior treatments, limit redosing due to small-scale individual production, and may not be effective if generated from dysfunctional $\mathrm{T}$ cells due to an immunosuppressive tumor microenvironment seen in many lymphoma types $[15,18,19]$. To overcome these issues related to cost, accessibility, efficacy, and efficiency, "off the shelf" allogeneic (allo) CAR-Ts are being explored. While they resolve some of the challenges encountered with autologous CAR-T, they also come with inherent hurdles such as the risk of graft versus host disease (GvHD) and rejection of the CAR-Ts by the host terminating their treatment effect $[20,21]$. We herein review the different strategies of generating, overcoming challenges associated with allo CAR-Ts, their clinical experience in lymphoma, and implications of their use.

\section{Strategies to generate "off the shelf" or universal allogeneic CAR-T}

For a successful generation of "off the shelf" or "universal" allo CAR-Ts, two important hurdles need solutions. First is the GvHD, which is mediated by the alloreactivity of the T cell receptor (TCR) on the CAR-T cells, which recognize the recipient's human leukocyte antigens (HLA) as foreign and mount an immune attack. Contrary to the first issue is the risk of rejection of allo CARTs by the recipient's immune system, resulting in their destruction. An essential requirement for the allo CAR-Ts to succeed is their persistence in the recipient, and lack thereof would not allow for sustained responses. While this does not apply to autologous CAR-Ts as seen in aggressive NHL with CD28 costimulatory domain in axi-cel, allo CAR-Ts are subject to rapid elimination by the host immune system, further shortening their persistence. Additionally, with allo CAR-Ts, there is also a risk for alloimmunization, which may lead to 
issues with redosing similar products due to donor-specific antibodies (DSA). Similar problems due to DSA have been encountered in patients undergoing solid organ transplantation and haploidentical stem cell transplantation [22, 23]. Comparison of features and implications between autologous and allogeneic CAR-T is outlined in Table 1. Several strategies to overcome these issues outlined above are in development and discussed here (Fig. 1).

\section{Developing CAR-T from a stem cell transplant donor}

$\alpha \beta$ T cells are the main drivers of GvHD in allogeneic stem cell transplant recipients $[24-26,27-29]$. One strategy to decrease the risk of GvHD for patients who have already received an allogeneic stem cell transplantation and have subsequently relapsed is to derive CAR-Ts from the same donor $\mathrm{T}$ cells. This was attempted in a clinical trial where 20 patients with B cell malignancies that had relapsed after allo stem cell transplant received a single infusion of donor-derived CAR-Ts targeting CD19 with no prior lymphodepletion chemotherapy. Eight of the 20 patients achieved a response with 6 complete remissions (CR). No GvHD was reported, and CAR-T expansion was higher for those with a

\section{Table 1. Differences between the autologous and allogenic CAR-Ts}

\begin{tabular}{|c|c|c|}
\hline Characteristic & Autologous & Allogeneic \\
\hline Cell source & Self & Non-self healthy donor, iPSC derived \\
\hline Production time & 2-5 weeks & Ready to use/off the shelf \\
\hline Cell type & $\begin{array}{l}\text { Autologous T cells (may or may not be manipulated } \\
\text { for T cell composition) }\end{array}$ & $\begin{array}{l}\text { Gene-edited } \alpha \beta \text { T cells, } \gamma \delta \text { T cells, NK cells, iNKT } \\
\text { cells }\end{array}$ \\
\hline $\begin{array}{l}\text { Manufacturing } \\
\text { process }\end{array}$ & $\begin{array}{l}\text { - Individualized manufacturing for each patient } \\
\text { - Reduced accessibility due to manufacturing wait } \\
\text { and potential impact of patients' native cells on } \\
\text { manufacturing success } \\
\text { - High variability in product composition due to } \\
\text { inherent patient heterogeneity in T cell composition } \\
\text { and immune profile }\end{array}$ & $\begin{array}{l}\text { - Single manufacturing from one donor or cell } \\
\text { source potentially used to treat many patients } \\
\text { - Pre-made and ready to use to remove the } \\
\text { manufacturing wait time } \\
\text { - Possible standardization and control over T cell } \\
\text { composition in the product }\end{array}$ \\
\hline Side Effects & $\begin{array}{l}\text { - Cytokine release syndrome } \\
\text { - Immune effector cell-associated neurotoxicity } \\
\text { syndrome } \\
\text { - B cell aplasia } \\
\text { - CAR gene editing-related oncogenic potential }\end{array}$ & $\begin{array}{l}\text { - Same as autologous PLUS } \\
\text { - More immune suppression and risk for infection } \\
\text { from more intense lymphodepletion for prevention } \\
\text { of allo rejection } \\
\text { - Graft versus host disease } \\
\text { - Allo rejection of the infusion product }\end{array}$ \\
\hline Redosing & $\begin{array}{l}\text { Response with the same CAR-T unfavorable in } \\
\text { aggressive lymphoma, possibly more promising in } \\
\text { FL } \\
\text { More studies needed to understand mechanisms of } \\
\text { relapse and resistance to redosing }\end{array}$ & $\begin{array}{l}\text { Benefit to be investigated } \\
\text { Risk of alloimmunization-donor-specific } \\
\text { antibodies }\end{array}$ \\
\hline Persistence & Months to years & Weeks to months \\
\hline Cost & High & Unknown (projected to be lower) \\
\hline
\end{tabular}




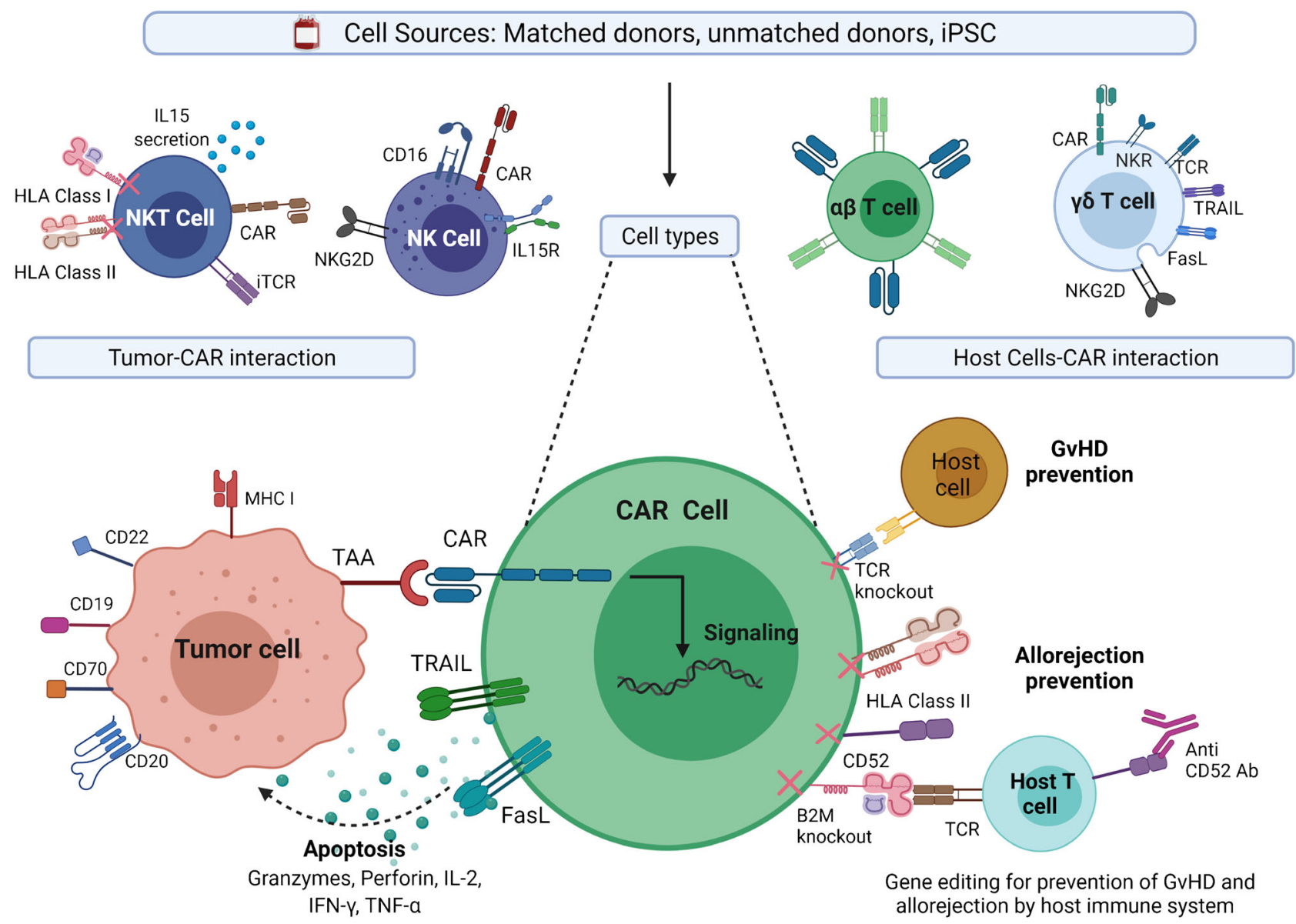

Fig. 1. Various allogeneic CARs with genomic editing techniques (abbreviations: CAR, chimeric antigen receptor; TAA, tumor associated antigen; B2M, $\beta 2$ microglobulin; Ab, antibody; TCR, T cell receptor; FasL, Fas ligand; iPSC, induced pluripotent stem cells; TRAIL, tumor necrosis factor-related apoptosis-inducing ligand; IL, interleukin; NKG2D, natural killer group 2 member D).

response than non-responders, and no CAR-T cells persisted beyond 3 weeks [30-32]. This study proved the feasibility, safety, and initial efficacy of such an approach albeit, with small patient numbers. Additionally, it highlights the use of allo CAR-Ts to generate a graft versus leukemia/lymphoma effect without significantly increasing the risk for GvHD. Another recent phase I clinical trial of 10 patients with relapsed or refractory B-acute lymphoblastic leukemia or aggressive lymphoma described the generation of CD19-targeting CAR-T from HLA-matched sibling donors using the high-capacity piggy-Bac transposon method for genetic modification. At a median follow-up of 18 months, 5 patients were in CR [33]. Interestingly, 2 of the 10 patients developed malignant lymphoma derived from CAR gene-modified $\mathrm{T}$ cells, and therefore, this strategy requires further follow-up [34]. It remains patient-specific and limited to those who have previously undergone allo stem cell transplant with available T cells and not with a history of GvHD. 


\section{Selecting non-alloreactive T cells as a source for CAR-T}

These have been used for several years now in patients with allo stem cell transplants for the treatment of viral infections without additional risk of GvHD [35-37]. This is thought to be due to the restricted repertoire of these memory T cells. Another disease where this approach has been promising is the Epstein-Barr virus (EBV)-associated malignancies, such as posttransplant associated lymphoproliferative disorder (PTLD). This approach combines the antigen specificity of CAR-Ts with the TCR specificity towards EBV. Recently, Prockop and colleagues reported on a single-center cohort of 46 patients with rituximab refractory PTLD developing after allo transplant $(n=33)$ or solid organ transplant $(n=13)$ who were treated with banked third-party partially HLA-matched EBV-specific cytotoxic T cells. Each treatment cycle consisted of 3 weekly infusions, followed by a 3-week observation period. This product named tabelecleucel showed a 1-year estimated overall survival (OS) for patients with PTLD following allo stem cell transplant and solid organ transplant of 68 and 64\%, respectively. The median OS for post allo transplant PTLD cohort had not been reached after a median follow-up of 23.3 months. The median OS for post solid organ transplant PTLD cohort was 21.3 months. Treatment-related serious adverse events were reported in 1 patient each in both the allo and solid organ transplant cohorts, respectively $[38,39,40]$. Tabelecleucel is currently in phase 2 (NCT04554914) and 3 trials for EBV+ PTLD following allo stem cell transplant post rituximab failure (NCT03392142, ALLELE study) and solid organ transplant after the failure of rituximab or rituximab and chemotherapy (NCT03394365).

These have a more restricted TCR repertoire as compared to naïve T cells and are therefore associated with less GvHD [41, 42]. They have shown to generate an effective anti-tumor response both in the preclinical and in autologous setting in non-Hodgkin lymphoma [43, 44]. However, the studies suggest a correlation between the presence of less-differentiated $\mathrm{T}$ cells, such as naïve $T$ cells $\left(T_{n}\right)$, central memory $T$ cells $\left(T_{c m}\right)$, and stem celllike memory $\mathrm{T}$ cells $\left(\mathrm{T}_{\mathrm{scm}}\right)$ with CAR-T efficacy. These less-differentiated $\mathrm{T}$ cell subsets are essential for in vivo expansion, survival, and persistence [45, $46,47]$. Various T cell subtypes exist with varied phenotypes and functions in the tumor microenvironment of lymphoma [18, 48, 49]. A T cell subset expressing CD8, CD45RA, and chemokine receptor 7 (CCR7) with features of $T_{n}$ cells or $T_{s c m}$ cells was found to be associated with increased in vivo expansion of CAR-T cells in lymphoma [50]. Fraietta et al. performed genomic, phenotypic, and functional testing to identify the determinants of response in CD19-targeting autologous CAR-T in chronic lymphocytic leukemia (CLL) and BCMA targeting autologous CAR-T in multiple myeloma. Sustained remission was associated with lymphocytes that possessed memory-like characteristics and had $\mathrm{CD} 27^{+} \mathrm{CD} 45 \mathrm{RO}^{-} \mathrm{CD} 8^{+}$expressing $\mathrm{T}$ cells before CAR-T generation [51, 52]. Therefore, further research is 


\section{Using genome editing of $\alpha \beta$ T cells}

required to better understand the ideal composition of various $\mathrm{T}$ cell subsets in the allo CAR-T product. Only selecting for $\mathrm{T}_{\mathrm{em}}$ cells might lead to ineffective therapy. No trials of allogeneic CAR-T with this strategy are currently ongoing.

Novel genome editing tools have paved the way to using conventional $\alpha \beta \mathrm{T}$ cells to generate allo CAR-T $[20,53,54] . \alpha \beta$ TCR removal by disruption of the gene encoding for alpha chain (TRAC) is sufficient and essential for preventing TCR-dependent GvHD. Torikai et al. were the first to report the feasibility and efficacy of $\alpha \beta$ TCR(neg) CD19CAR(pos) T cells using the zinc finger nucleases (ZFNs) for permanent deletion of $\alpha$ or $\beta$ TCR chains in B cell malignancies [55•]. Another genome editing tool used by Poirot et al. transcription activatorlike effector nuclease (TALEN) was able to perform multiplex gene editing to manufacture T cells deficient in expression of both $\alpha \beta$ TCR and CD52 [56]. These T cells were unable to cause GvHD in a mouse model and were resistant to removal by alemtuzumab (anti-CD52 monoclonal antibody), which can be used to eliminate host $\mathrm{T}$ cells and hence avoid rejection. Cluster regularly interspaced short palindromic repeats (CRISPR)/Cas9 with its rapid and efficient multiplex genome editing have been recently used to generate allogeneic universal CAR-T with up to 4 disrupted genes $[57,58]$. While these approaches are very promising, they are not free from consequences. They may not result in complete knock out of $\alpha \beta$ TCR in all T cells resulting in GvHD [59]. Two, there is a risk of "off-target" genome cleavage resulting in unwanted gene translocations or inactivation that could potentially confer oncogenic potential on the CAR-Ts [56]. Three, these multiple genomic changes could result in increased toxicity, poor transduction efficacy, or depending on the gene disruption, a proliferation and survival advantage to the CAR-Ts [60].

Recently, CRISPR/Cas9 technology was used to integrate CAR construct into the TRAC locus, to ensure that TCR is inactivated at the same time as CAR introduction $[61 \bullet, 62]$. Other advantages of this approach are that it is safer without insertional mutagenesis-related adverse effects. The CAR expression is regulated by endogenous TCR promoter, preventing constant $\mathrm{T}$ cell activation, differentiation, and exhaustion. These advantages render more potent antitumor activity compared to the conventionally transduced CAR-Ts $[61 \bullet, 63$, 64]. UCART19 an allo CD19 CAR-T is currently under evaluation in adult and pediatric ALL with promising result and up to $50 \%$ of patients able to proceed to allogeneic transplant [65].

There is still the risk of allo-rejection with TCR-negative allogeneic CAR-Ts due to the recognition of non-self HLA by the recipient's T cells if there is a mismatch. While strategies such as lymphodepletion and irradiation can help delay the rejection, the CAR-T persistence which is necessary for efficacy will be relatively short. For this, the removal of class I HLA molecules is required from the $\mathrm{T}$ cells, which can be achieved by disrupting the beta- 2 microglobulin $(\beta 2 \mathrm{M})$ gene locus [57, 66-68]. CD19 and CD70 (COBALT-LYM)-targeting CAR-Ts with CRISPR/Cas9 disrupted TRAC and $\beta 2 \mathrm{M}$ genes (double knockout) are currently under evaluation in B cell lymphomas and leukemias (NCT03166878, NCT04502446). Further triple knockout of HLA classes I and II and TRAC by CRISPR-based techniques has shown even better persistence of CAR-Ts in vivo when compared to double knockout [67]. While the HLA class I 
knockout prevents allo-rejection from T cells, it does not contain the allo CARTs from potential rejection by NK cells. Avoiding recognition by NK cells can be achieved by expression of non-classical HLA molecules such as HLA-E and G $[66,69]$. The use of HLA homozygous donors to generate a bank of universal allo CARs can also help prevent rejection due to HLA mismatch [70]. Genome editing techniques indeed open up a wide range of opportunities in the development of allo CAR-Ts; clinical studies are needed to determine their efficacy and safety for use.

\section{Using non- $\alpha \beta$ TCR T cells for CAR approach}

Several other immune cell types can generate CAR-based therapy if they possess cytotoxic properties and are easily accessible from sources such as peripheral blood mononuclear cells (PMBCs), or renewable stem cells.

Natural killer (NK) cells are an attractive alternative as they are highly cytolytic to cancer cells via granzyme B, perforin, and Fas ligand. They are an integral part of tumor immunosurveillance and are often found to be dysfunctional due to cancer's immunosuppressive mechanisms for immune escape. Due to their innate anti-tumor activity, without causing GvHD, CAR-expressing NK is appealing. This has been shown to be feasible and active in in vitro and xenogeneic tumor models, as well as against patient-derived glioblastoma cell lines [71, 72]. However, NK cells exist in low numbers in peripheral blood and are less pliable to genetic manipulation which makes its use difficult [73, 74]. A strategy uses the NK92 cell line, an activated human NK cell line derived from an NHL patient [75, 76]. CAR-NK cells derived from the NK92 cell line targeting CD20 have shown efficacy against lymphomas in vitro [77]. NK-92 cell line has disadvantages as it is derived from a patient with NK lymphoma and has the potential for tumor engraftment post infusion. In addition, these cells are EBV positive, which carry various cytogenetic abnormalities. Due to these reasons, the NK92 cell line needs to be irradiated before infusion into patients, which can negatively impact its expansion and persistence, ultimately leading to low efficacy [78]. Another strategy using umbilical cord blood (UCB) to obtain NK cells has been used to create CD19 directed CAR-NK cells. These UCB-derived NK cells were transduced with a retroviral vector incorporating the genes for CAR-CD19, interleukin-15 (IL-15), and inducible caspase-9-based suicide gene (iCasp9). Liu et al. reported efficient killing of CD19-expressing cell lines, with marked prolongation of survival in a xenograft lymphoma murine model. IL-15 production by the transduced UCB-NK cells critically improved their function over controls. The iCasp9 suicide gene upon pharmacologic activation resulted in rapid elimination of the iCasp9/CAR.19/IL-15 UCB-NK cells, thereby incorporating a safety switch [79]. This approach was evaluated in a phase I/II clinical trial where HLA-mismatched UCB-derived CAR-NK cells targeting CD19 were infused in 11 patients with relapsed or refractory CD19-positive B cell NHL or CLL. There were no cytokine release syndrome (CRS), neurotoxicity, or GvHD, and no increase in inflammatory markers such as IL-6. Of the 11 patients, 8 $(73 \%)$ had a response, with seven patients achieving a CR; however, the full clinical activities of the NK cells were difficult to interpret as many patients received subsequent systemic therapy or stem cell transplant as consolidation/ maintenance. The CAR-NK cells were detectable in some subjects at low levels for up to 12 months post infusion [80•]. To overcome the limited amount of 
NK cell present even in the UCB, a Good Manufacturing Practice (GMP)compliant procedure has been developed which reliably generates clinically relevant doses of GMP-grade NK cells from a UCB unit [81]. These studies support the use of NK cells as a source of allo CAR-Ts, and several products are currently in early phase clinical trials (Table 2 ).

Induced pluripotent stem cells (iPSCs) are another source for CARs. These can be genetically modified at a clonal level and used to create a homogeneous population of uniformly engineered T or NK cells. These have certain advantages, such as their unlimited division potential, and are more amenable to genetic modification [82]. Any somatic cell can be reprogrammed into iPSC; single clones from these cells can be expanded to create an iPSC cell line. T cells generated from iPSC still need genetic editing to prevent GvHD; however, once modified, a single cell can then be used to generate a clonal population of CARTs with desired knockouts. Clinical trials are already underway using iPSCderived CAR-Ts and CAR-NK cells in B cell lymphomas (NCT04023071, NCT04245722, NCT04629729; Table 2) [83].

NK T (NKT) cells are a subset of T cells that express NK cell markers. A subset of NKT cells called the "invariant NKT" (iNKT) cells expressed a highly restricted TCR, which can recognize lipid antigens presented by CD1d (HLA class I-like molecule) on B cells, antigen-presenting cells, and some epithelial cells [84, 85]. Allogeneic or donor iNKT cells have also shown to be protective against GvHD due to the production of IL- 4 and promotion of a Th2-based immune response in preclinical models and clinical acute GvHD [86-88]. CD19-targeting CARiNKT cells showed a significantly improved efficacy over conventional CD19 CAR-Ts in a murine model of CD1d+CD19+ B cell malignancy [89]. Protective effect against GvHD and improved efficacy over conventional CD19 CAR-Ts make this an explorable approach which is currently in phase I trial in B cell malignancies (NCT03774654).

$\gamma \delta \mathrm{T}$ cells are another potential candidate under investigation as allogeneic CAR-Ts. They represent a small percentage of circulating lymphocytes (1-5\%), however, in abundance in certain tissue sites such as gut mucosa, reproductive organs, tongue, and skin [90-92]. $\gamma \delta$ T cells form a key component of the innate immune system and tumor immunosurveillance and can target tumor antigens not recognized by $\alpha \beta$ T cells [93]. Additionally, their tissue residency gives them an advantage in efficacy over $\alpha \beta$ CAR-Ts which have poor penetrance into noninflamed tumors. They are also less likely to induce GvHD as their TCR activation is not MHC restricted [94]. CD19-targeting CAR-T developed from $\gamma \delta$ T cells has already shown efficacy in CD19+ B cell malignancies in vitro and in vivo and also in an early phase clinical trial (NCT02656147) [95].

\section{Clinical experience with allogeneic CAR-T in lymphoma}

A list of completed and ongoing clinical trials using allo CAR-Ts in lymphoma is shown in Table 2. ALLO-501 is a genetically modified anti-CD19 CAR-T in which the TCR $\alpha$ gene is disrupted to reduce the risk of GvHD, and the CD52 gene is disrupted to permit the use of ALLO-647 an anti-CD52 monoclonal antibody ( $\mathrm{mAb}$ ), for selective and prolonged host lymphodepletion. The phase I ALPHA study (NCT03939026) was first presented at the ASCO 2020 meeting. This included patients with R/R LBCL and follicular lymphoma (FL) after two or 
more lines of therapy. Prior exposure to CD19-directed therapy was allowed. The data were presented for 22 patients with age range $34-73$ years, $64 \%$ with LBCL, and $41 \%$ with prior autologous stem cell transplant, and $18 \%$ with autologous CAR-T. The overall response rate (ORR) was 63\%, with a 37\% complete remission rate (CR) in the 19 evaluable patients. Retreatment was noted to be feasible. The most common grade $\geq 3$ adverse events (AEs) were cytopenias. CRS occurred in 32\% (7 patients), and none with grade $\geq 3$; no doselimiting toxicities or GvHD was observed [96•,97]. This was further updated for 42 patients (infused 41; $11 \mathrm{LBCL}$, and $21 \mathrm{FL}$ ) at the ASCO 2021 meeting, including 9 patients that had previously received autologous CAR-T. The median time from enrollment to start of therapy was 5 days in $98 \%$ of the enrolled patients. The efficacy analysis was available for 32 patients, and ORR and CR rates were 75\% (24) and 50\% (16), respectively. Redosing led to clinical responses, with an overall treatment failure-free survival for autologous CAR-T naïve patients of $64 \%$ and $61 \%$ at 6 months for FL and LBCL. No dose-limiting toxicities or GvHD was observed, and one grade 3 neurotoxicity was reported. CRS occurred in $27 \%$ of patients, and none with grade $\geq 3$. There were 5 treatment-emergent deaths, one each from pneumonia, arrhythmia, stroke, and two instances of COVID-19 infection. The ALPHA2 study (NCT04416984), an open-label, single-arm study of ALLO-501A in non-HLA, matched patients with R/R LBCL after two or more lines of treatment also presented at the ASCO 2021 meeting. Prior autologous CD19 CAR-T was allowed if the tumor remained CD19+. As of the April 19th, 2021 data cutoff, 13 patients were enrolled, and 12 patients were treated with ALLO-501A. One patient that developed central nervous system involvement before infusion was not treated. Nine patients evaluable for efficacy were CAR-T naïve, except for one who received prior autologous CAR-T and previously had a 16-week CR followed by relapse. Of the 9 patients eligible for efficacy analysis ( $n=5$, in consolidation cohort), both the ORR and CR rates were $56 \%$. No dose-limiting toxicities, GvHD, or neurotoxicity was seen, and CRS occurred in 2 patients, both grade $<3$ [98].

PBCAR0191, an off-the-shelf, allogeneic CAR-T product, is also being evaluated in the CD19+ R/R B cell NHL (NCT03666000). The preliminary safety and efficacy data from the ASCO 2021 meeting on 13 patients have been presented. The median time from eligibility confirmation to infusion was 6.5 days. No grade $\geq 3$ CRS or neurotoxicity was observed, and no GvHD was noted either. The ORR at day+28 was $77 \%$ with a $54 \%$ CR rate [99]. The safety and efficacy of CTX110, an allo anti-CD19 CAR-T cell product modified by using CRISPR/Cas9 editing to disrupt the endogenous TRAC locus and disrupt $\beta_{2} \mathrm{M}$, which eliminates major histocompatibility complex (MHC) class I expression, are under evaluation in R/R LBCL, double/triple hit lymphoma, transformed FL, and FL grade 3b. The phase 1 CARBON trial (NCT04035434) is an open-label, multicenter, global study enrolling patients with 2 or more lines of treatment. Prior allogeneic stem cell transplant and autologous CD19 CAR-T are excluded. This trial is open and enrolling at this time, and the data are awaited [100].

\section{Implications of allogeneic CAR-T}

Allogeneic "off the shelf" CAR-T offers the promise of a readily available, hopefully at a reduced cost and more accessible to the patients and resource- 


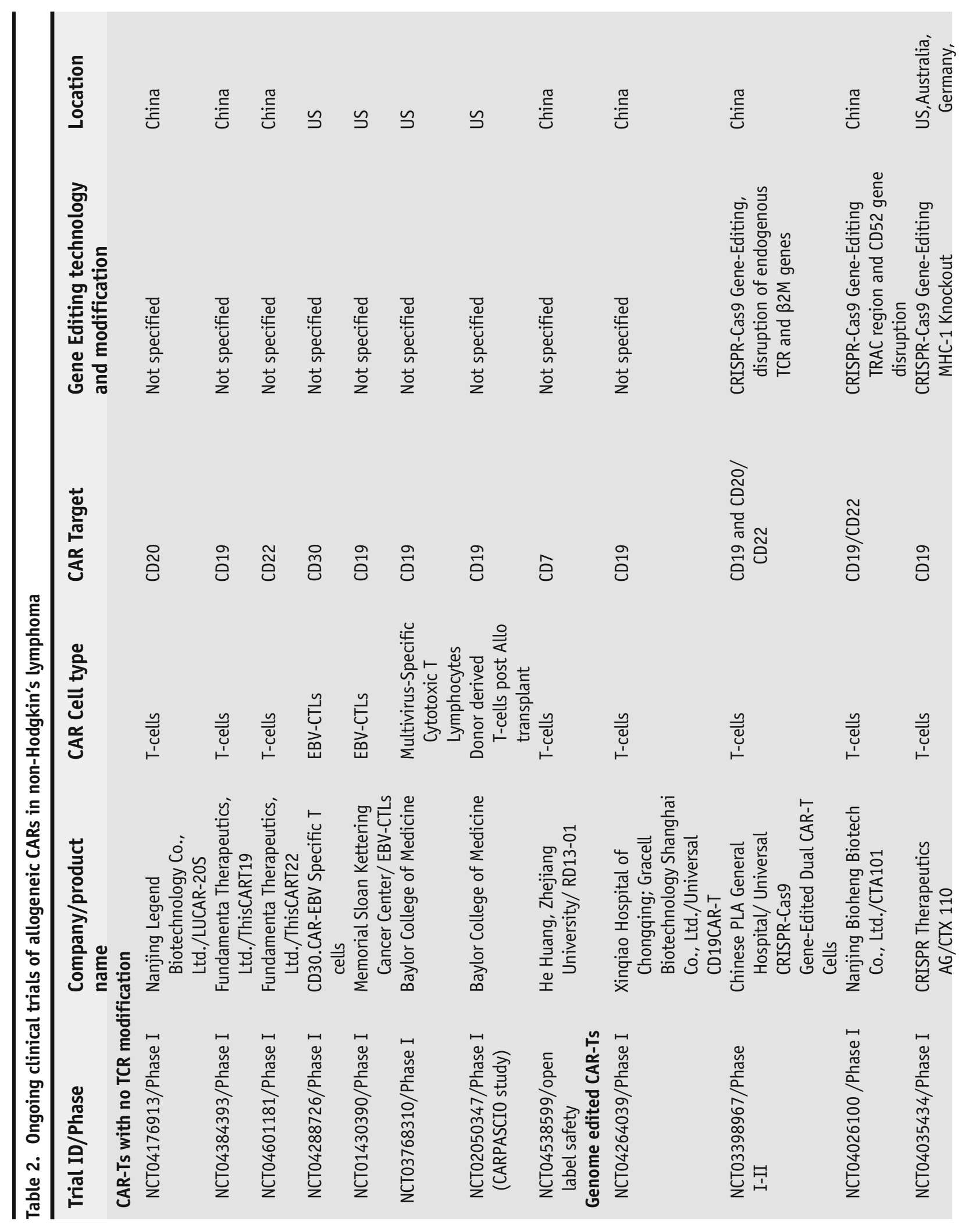




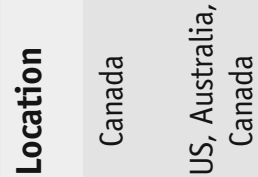

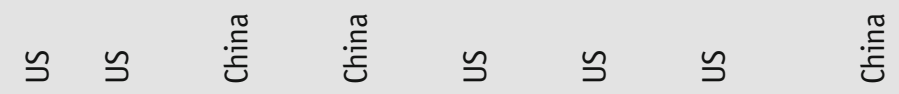

$\cong$

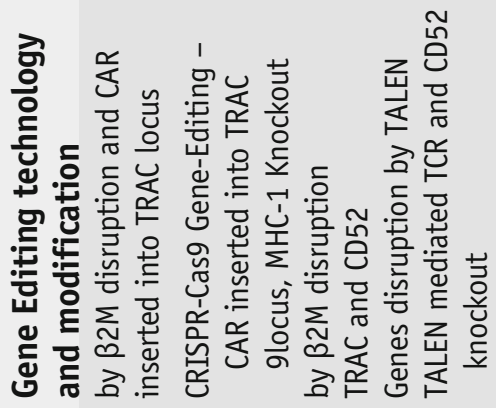

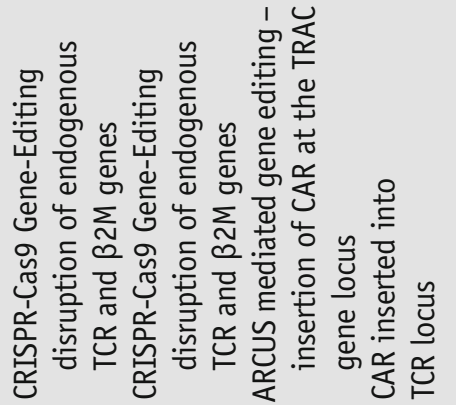

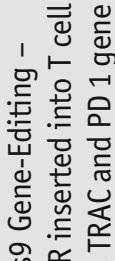

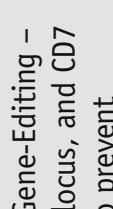

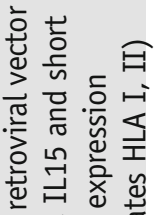

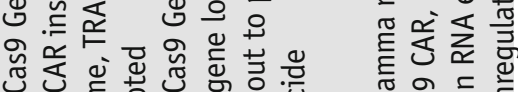

일

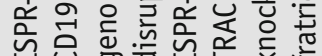

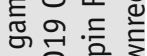

尊记

ڤ

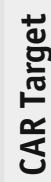

옹

ปे

อิ

อั่

ปิ) Оิ

ํํㅁ

อิ

อे

齐

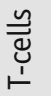

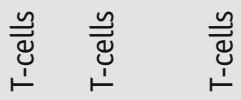

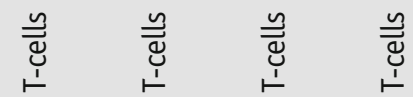

$\underset{\substack{\tilde{Z} \\ 亡}}{\stackrel{y}{1}}$

$\stackrel{y}{0}$
$\frac{5}{z}$

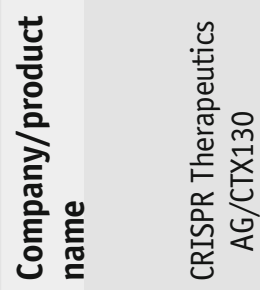

웅

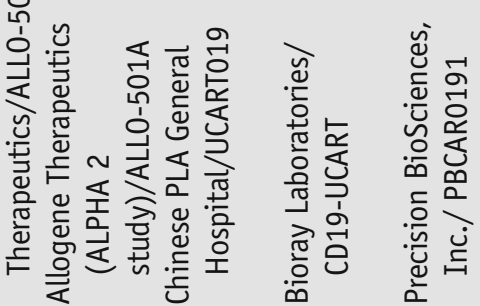

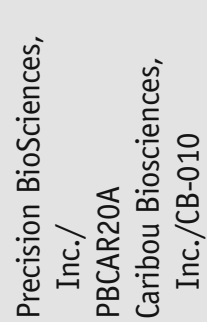

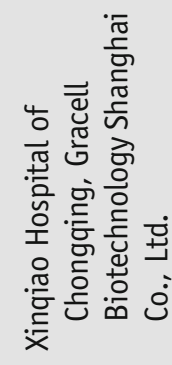

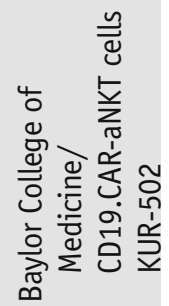

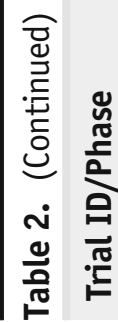

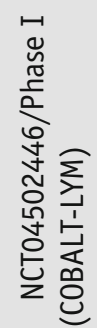

밀

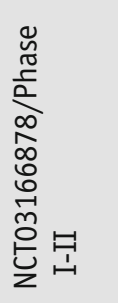

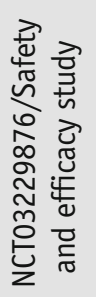

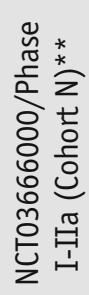

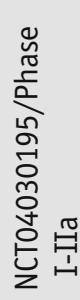

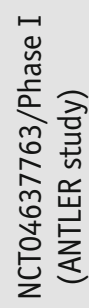

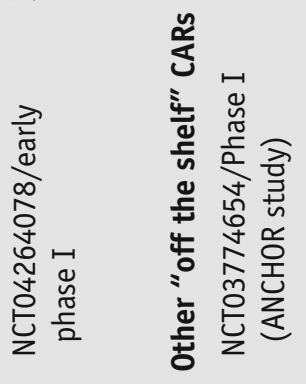




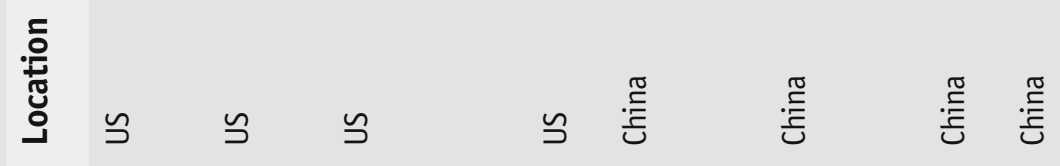

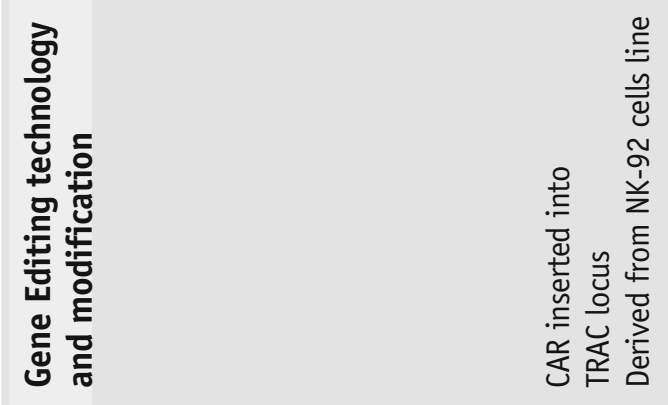

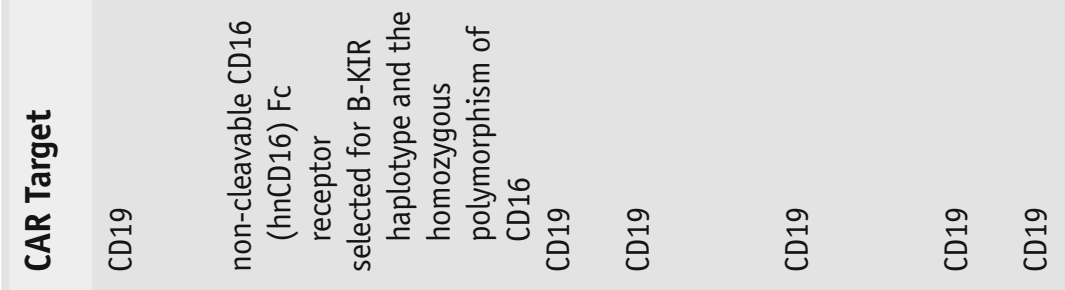

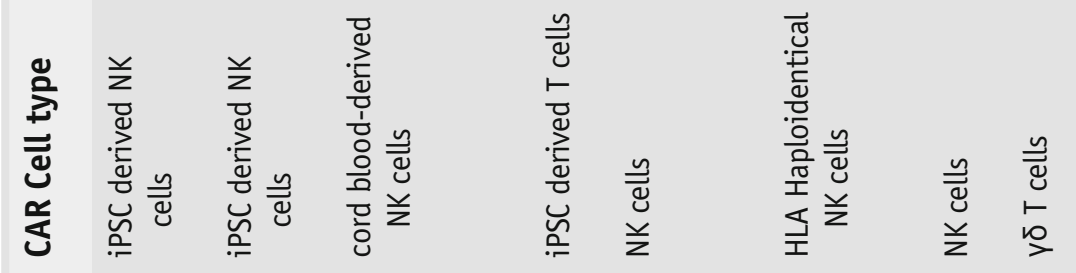

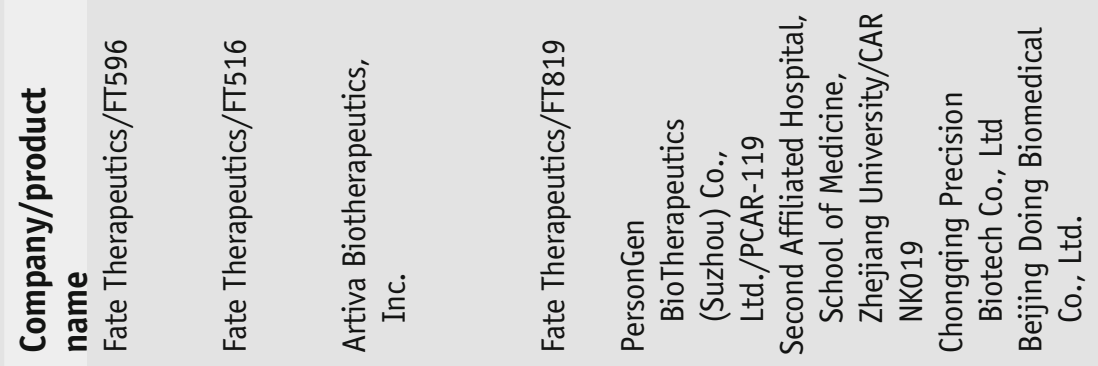

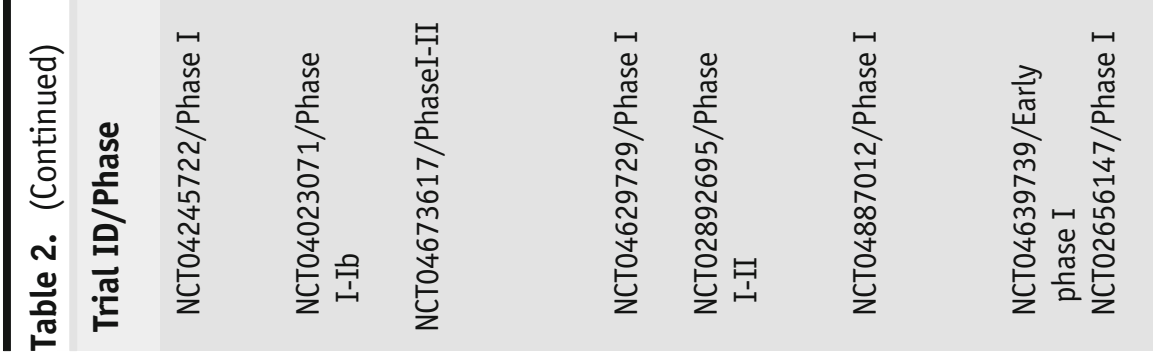


limited settings. In addition, the ability to generate CAR-Ts from healthy donor cells with better quality without exposure to prior chemotherapies or the immunosuppressive NHL microenvironment could be transformative. It would also allow for redosing and standardized scale production and quality maintenance. These could also permit targeting multiple antigens simultaneously or sequentially. Genome editing tools enable a broader application of these "off the shelf" products with multiplex gene knockout and targeted transductions focused on making them safer (less GvHD) and persistent (less allo rejection) for effective use. Still, many challenges remain in enhancing the efficacy of allo CAR-Ts, such as the degree of in vivo expansion and persistence of CAR-Ts for efficacy and effective lymphodepletion strategies, which is likely of greater importance in this setting. Also, the impact of allo CAR-T persistence on efficacy is yet to be determined as these are more likely to be eliminated by the recipient's immune system. Redosing with the same or another allo CAR targeting different antigens may be the solution, carefully considering donorspecific antibodies. The association of persistence of CAR-T cells in aggressive lymphoma is still debatable, as autologous CAR-T with CD28 signaling domain (axi-cel) with shorter persistence can still ensue durable responses in aggressive lymphomas. On the other hand, redosing with the axi-cel in indolent lymphoma such as FL or marginal zone lymphoma (MZL) as done in the ZUMA-5 trial in 13 patients ( $11 \mathrm{FL}, 2 \mathrm{MZL}$ ) showed an ORR rate of $100 \%$ and a $77 \%$ CR rate. The median duration of response after redosing had not been reached, and 8 and 4 patients developed CRS and neurotoxicity, respectively but no grade $\geq 3$ [101]. Lastly, determination of the optimal subset of T cells, significantly enriched with less differentiated naïve, and stem cell-like memory cells would be critical for optimal expansion, survival, and long-term persistence. As more clinical data are generated by bringing these promising therapies to the bedside for efficacy and side effects, further advances will be made to create an ideal "off the shelf" CAR-T product.

\section{Conclusions}

CAR-T has revolutionized the treatment landscape of lymphomas and other cancers. Developing low-cost, readily available, easily accessible, safe, and effective products using genome editing tools and non-alloreactive cells will help generate true "off the shelf products." With the number of active investigations, more transformative updates are anticipated soon to advance the capacity of this novel therapy.

\section{Declarations}

\section{Conflict of Interest}

Arushi Khurana declares that she has no conflict of interest. Yi Lin has received research funding (paid to Mayo Clinic) from Kite/Gilead, Celgene/Bristol-Myers Squibb, bluebird bio, Janssen, Legend Biotech, Takeda, Merck, and Boston Scientific; has served as a consultant for Kite/Gilead, Celgene/Bristol-Myers Squibb, Juno/Bristol-Myers Squibb, Janssen, Legend Biotech, Gamida Cell Ltd, Novartis, Iovance Biotherapeutics, Takeda, and Fosun Kite 
Biotechnology Co., Ltd; and has served on a Data Safety Monitoring Board (DSMB) for Sorrento Therapeutics. All funds were paid directly to Mayo Clinic. Dr. Lin received no personal compensation.

\section{Open Access}

This article is licensed under a Creative Commons Attribution 4.0 International License, which permits use, sharing, adaptation, distribution and reproduction in any medium or format, as long as you give appropriate credit to the original author(s) and the source, provide a link to the Creative Commons licence, and indicate if changes were made. The images or other third party material in this article are included in the article's Creative Commons licence, unless indicated otherwise in a credit line to the material. If material is not included in the article's Creative Commons licence and your intended use is not permitted by statutory regulation or exceeds the permitted use, you will need to obtain permission directly from the copyright holder. To view a copy of this licence, visit http://creativecommons.org/licenses/by/4.0/.

\section{References and Recommended Reading}

Papers of particular interest, published recently, have been highlighted as:

- $\quad$ Of importance

1. Crump M, et al. Outcomes in refractory diffuse large Bcell lymphoma: results from the international SCHOLAR-1 study. Blood. 2017;130:1800-8.

2. $\quad$ Neelapu SS, et al. Axicabtagene ciloleucel CAR T-cell therapy in refractory large B-cell lymphomA. N Engl J Med. 2017;377:2531-4.

First landmark registration trial for the CAR-T product axicabtagene ciloleucel that resulted in its approval for relapsed/refractory aggressive non-Hodgkin lymphoma.

3. $\quad$ Schuster SJ, et al. Chimeric antigen receptor T cells in refractory B-cell lymphomas. N Engl J Med. 2017;377:2545-5.

Landmark registration trial for the CAR-T product tisagenlecleucel that resulted in its approval for relapsed/refractory aggressive non-Hodgkin lymphoma.

4. $\quad$ Abramson JS, et al. Lisocabtagene maraleucel for patients with relapsed or refractory large B-cell lymphomas (TRANSCEND NHL 001): a multicentre seamless design study. Lancet. 2020;396:839-5.

Landmark registration trial for the CAR-T product

lisocabtagene maraleucel that resulted in its approval for relapsed/refractory aggressive non-Hodgkin lymphoma.

5. Locke FL, et al. Long-term safety and activity of axicabtagene ciloleucel in refractory large B-cell lymphoma (ZUMA-1): a single-arm, multicentre, phase 12 trial. Lancet Oncol. 2019;20:31-42.

6. $\quad$ Nastoupil LJ, et al. Standard-of-care axicabtagene ciloleucel for relapsed or refractory large B-cell lymphoma: results from the US lymphoma CAR T consortium. J Clin Oncol. 2020;38:3119-2.

First real-world evidence of use of axicabtagene ciloleucel including patients who would not have been eligible for ZUMA1 clinical trial.

7. Pasquini MC, et al. Post-marketing use outcomes of an anti-CD19 chimeric antigen receptor (CAR) T cell therapy, axicabtagene ciloleucel (Axi-Cel), for the

8. Riedell PA, et al. A multicenter retrospective analysis of treatment of large B cell lymphoma (LBCL) in the United States (US). Blood. 2019;134:764. clinical outcomes, toxicities, and patterns of use in institutions utilizing commercial axicabtagene ciloleucel and tisagenlecleucel for relapsed/refractory aggressive B-cell lymphomas. Blood. 2019;134:-1599.

9. Jacobson CA, et al. Axicabtagene ciloleucel in the nontrial setting: outcomes and correlates of response, resistance, and toxicity. J Clin Oncol. 2020;38:3095-106.

10. Jacobson CA, et al. Interim analysis of ZUMA-5: a phase II study of axicabtagene ciloleucel (axi-cel) in patients (pts) with relapsed/refractory indolent nonHodgkin lymphoma (R/R iNHL). J Clin Oncol. 2020;38:8008.

11. Wang M, et al. KTE-X19 CAR T-cell therapy in relapsed or refractory mantle-cell lymphoma. $N$ Engl J Med. 2020;382:1331-4.

Landmark registration trial for the CAR-T product brexucabtagene autoleucel that resulted in its approval for relapsed/refractory Mantle Cell lymphoma.

12. June $\mathrm{CH}$, Sadelain $\mathrm{M}$. Chimeric antigen receptor therapy. N Engl J Med. 2018;379:64-73.

13. Schuster SJ, et al. Tisagenlecleucel in adult relapsed or refractory diffuse large B-cell lymphoma. $N$ Engl J Med. 2019;380:45-56.

14. Pinnix CC, et al. Bridging therapy prior to axicabtagene ciloleucel for relapsed/refractory large B-cell lymphoma. Blood Adv. 2020;4:2871-83.

15. Locke FL, et al. Tumor burden, inflammation, and product attributes determine outcomes of axicabtagene ciloleucel in large B-cell lymphoma. Blood Adv. 2020;4:4898-911.

16. Khurana A et al. Lines of therapy before autologous stem cell transplant (ASCT) and CAR-T infusion affect outcomes in aggressive non-Hodgkin's lymphoma (NHL). 2021. 
17. Tully S et al. Impact of increasing wait times on overall mortality of chimeric antigen receptor T-cell therapy in large B-cell lymphoma: a discrete event simulation model. JCO Clin Cancer Inform 2019;1-9. https://doi. org/10.1200/cci.19.00086.

18. Khurana A, Ansell SM. Role of microenvironment in non-hodgkin lymphoma: understanding the composition and biology. Cancer Journal (United States). 2020;26:206-16.

19. Byrne $\mathrm{M}$, et al. Understanding and managing large $B$ cell lymphoma relapses after chimeric antigen receptor T cell therapy. Biology of Blood and Marrow Transplantation. 2019;25:e344-51.

20. Depil S, Duchateau P, Grupp SA, Mufti G, Poirot L. 'Off-the-shelf' allogeneic CAR T cells: development and challenges. Nature Reviews Drug Discovery. 2020;19:185-99.

21. Ruella M, Kenderian SS. Next-generation chimeric antigen receptor T-cell therapy: going off the shelf. BioDrugs. 2017;31:473-81.

22. Butler CL, Valenzuela NM, Thomas KA, Reed EF. Not all antibodies are created equal: factors that influence antibody mediated rejection. J Immunol Res $2017 ; 2017$

23. Ciurea SO, et al. The European Society for Blood and Marrow Transplantation (EBMT) consensus guidelines for the detection and treatment of donor-specific antiHLA antibodies (DSA) in haploidentical hematopoietic cell transplantation. Bone Marrow Transplantation. 2018;53:521-34.

24. Zeiser R, Blazar BR. Acute graft-versus-host disease-biologic process, prevention, and therapy. $N$ Engl J Med. 2017;377:2167-79.

25. Zeiser R, Blazar BR. Pathophysiology of chronic graftversus-host disease and therapeutic targets. N Engl J Med. 2017;377:2565-79.

26. Abdelhakim H, Abdel-Azim H, Saad A. Role of $\alpha \beta \mathrm{T}$ cell depletion in prevention of graft versus host disease. Biomedicines. 2017;5(3):35.

27. Van Rood JJ, et al. Effect of tolerance to noninherited maternal antigens on the occurrence of graft-versushost disease after bone marrow transplantation from a parent or an HLA-haploidentical sibling. Blood. 2002;99:1572-7.

28. Rådestad E et al. Individualization of hematopoietic stem cell transplantation using alpha/beta T-cell depletion. Front Immunol 2019;10.

29. Rådestad E et al. Alpha/beta T-cell depleted grafts as an immunological booster to treat graft failure after hematopoietic stem cell transplantation with HLAmatched related and unrelated donors. J Immunol Res 2014;2014.

30. Brudno JN, et al. Allogeneic T cells that express an antiCD19 chimeric antigen receptor induce remissions of B-cell malignancies that progress after allogeneic hematopoietic stem-cell transplantation without causing graft-versus-host disease. J Clin Oncol. 2016;34:111221.
31. Kochenderfer JN, et al. Donor-derived CD19-targeted T cells cause regression of malignancy persisting after allogeneic hematopoietic stem cell transplantation. Blood. 2013;122:4129-39.

32. Cruz CRY, et al. Infusion of donor-derived CD19redirected virus-specific $\mathrm{T}$ cells for B-cell malignancies relapsed after allogeneic stem cell transplant: a phase 1 study. Blood. 2013;122:2956-73.

33. Bishop DC, et al. Development of CAR T-cell lymphoma in two of ten patients effectively treated with piggyBac modified CD19 CAR T-cells. Blood. 2021. https://doi.org/10.1182/blood.2021010813.

34. Micklethwaite KP, et al. Investigation of product derived lymphoma following infusion of piggyBac modified CD19 chimeric antigen receptor T-cells. Blood. 2021. https://doi.org/10.1182/blood.2021010858.

35. Leen AM, et al. Multicenter study of banked third-party virus-specific T cells to treat severe viral infections after hematopoietic stem cell transplantation. Blood. 2013;121:5113-23.

36. Hanley PJ, et al. Functionally active virus-specific T cells that target $\mathrm{CMV}$, adenovirus, and EBV can be expanded from naive T-cell populations in cord blood and will target a range of viral epitopes. Blood. 2009;114:195867.

37. Melenhorst JJ, et al. Allogeneic virus-specific T cells with HLA alloreactivity do not produce GVHD in human subjects. Blood. 2010;116:4700-2.

38. Long term outcomes of tabelecleucel (allogeneic thirdparty.... EHA Library. Prockop S. Jun 15 2018; 214869. https://library.ehaweb.org/eha/2018/stockholm/ 214869/susan.prockop.long.term.outcomes.of. tabelecleucel.28allogeneic.third-party.html?f=listing\% 3D4\%2Abrowseby\%3D8\%2Asortby $\% 3 \mathrm{D} 2 \%$ 2Amedia\%3D3\%2Aspeaker\%3D664306.

39. O'Reilly RJ, Prockop S, Hasan AN, Koehne G, Doubrovina E. Virus-specific T-cell banks for 'off the shelf' adoptive therapy of refractory infections. Bone Marrow Transplantation. 2016;51:1163-72.

40. Prockop S, et al. Off-the-shelf EBV-specific T cell immunotherapy for rituximab-refractory EBV-associated lymphoma following transplantation. J Clin Invest. 2020;130:733-47.

41. Robins HS, et al. Comprehensive assessment of T-cell receptor $\beta$-chain diversity in $\alpha \beta$ T cells. Blood. 2009;114:4099-107.

42. Foster AE, et al. Human CD62L-memory T cells are less responsive to alloantigen stimulation than $\mathrm{CD} 62 \mathrm{~L}+$ naive T cells: potential for adoptive immunotherapy and allodepletion. Blood. 2004;104:2403-9.

43. Turtle CJ et al. Immunotherapy of non-Hodgkin's lymphoma with a defined ratio of CD8+ and CD4+ CD19-specific chimeric antigen receptor-modified $\mathrm{T}$ cells. Sci Transl Med 2016;8.

44. Chan WK, et al. Chimeric antigen receptor-redirected CD45RA-negative T cells have potent antileukemia and pathogen memory response without graft-versus-host activity. Leukemia. 2015;29:387-95. 
45. Berger C, et al. Adoptive transfer of effector CD8+ T cells derived from central memory cells establishes persistent T cell memory in primates. J Clin Invest. 2008;118:294-305.

46. Gattinoni L, et al. A human memory T cell subset with stem cell-like properties. Nat Med. 2011;17:1290-7.

47. Sommermeyer D, et al. Chimeric antigen receptormodified T cells derived from defined CD8+ and CD4+ subsets confer superior anti-tumor reactivity in vivo. Leukemia. 2016;30:492-500.

48. Tarte K. Role of the microenvironment across histological subtypes of NHL. Hematology. 2017;2017:6107.

49. Ansell SM, Vonderheide RH. Cellular composition of the tumor microenvironment. Am Soc Clin Oncol Educ B. 2013;33:e91-7.

50. Xu Y, et al. Closely related T-memory stem cells correlate with in vivo expansion of CAR.CD19-T cells and are preserved by IL-7 and IL-15. Blood. 2014;123:37509.

51. Fraietta JA, et al. Determinants of response and resistance to CD19 chimeric antigen receptor (CAR) T cell therapy of chronic lymphocytic leukemia. Nat Med. 2018;24:563-71.

52. Garfall AL, et al. T-cell phenotypes associated with effective CAR T-cell therapy in postinduction vs relapsed multiple myeloma. Blood Adv. 2019;3:2812-5.

53. Manriquez-Roman C, Siegler EL, Kenderian SS. CRISPR takes the front seat in CART-cell development. BioDrugs. 2021;35:113-24.

54. Yin H, Kauffman KJ, Anderson DG. Delivery technologies for genome editing. Nature Reviews Drug Discovery. 2017;16:387-99.

55. Torikai H, et al. A foundation for universal T-cell based immunotherapy: T cells engineered to express a CD19specific chimeric-antigen-receptor and eliminate expression of endogenous TCR. Blood. 2012;119:569770.

This is the first study describing the development of allogeneic universal CAR T cells using gene editing by eliminating TCR as a way to avoid GvHD.

56. Philip LPB, et al. Multiplex genome-edited T-cell manufacturing platform for 'off-the-shelf' adoptive Tcell immunotherapies. Cancer Res. 2015;75:3853-64.

57. Ren J, et al. Multiplex genome editing to generate universal CAR T cells resistant to PD1 inhibition. Clin Cancer Res. 2017;23:2255-66.

58. Ren J, et al. A versatile system for rapid multiplex genome-edited CAR T cell generation. Oncotarget. 2017;8:17002-11.

59. Qasim W et al. Molecular remission of infant B-ALL after infusion of universal TALEN gene-edited CAR T cells. Sci Transl Med 2017;9.

60. Fraietta JA, et al. Disruption of TET2 promotes the therapeutic efficacy of CD19-targeted T cells. Nature. 2018;558:307-12.

61. Eyquem J, et al. Targeting a CAR to the TRAC locus with CRISPR/Cas9 enhances tumour rejection. Nature. 2017;543:113-.
This study shows the advantages of targeting the CAR directly to the TRAC locus.

62. MacLeod DT, et al. Integration of a CD19 CAR into the TCR alpha chain locus streamlines production of allogeneic gene-edited CAR T cells. Mol. Ther. 2017;25:949-61.

63. Hale $\mathrm{M}$, et al. Homology-directed recombination for enhanced engineering of chimeric antigen receptor $\mathrm{T}$ cells. Mol Ther Methods Clin Dev. 2017;4:192-203.

64. Georgiadis $\mathrm{C}$, et al. Long terminal repeat CRISPR-CARcoupled "universal" $\mathrm{T}$ cells mediate potent antileukemic effects. Mol Ther. 2018;26:1215-27.

65. Benjamin $\mathrm{R}$, et al. Genome-edited, donor-derived allogeneic anti-CD19 chimeric antigen receptor T cells in paediatric and adult B-cell acute lymphoblastic leukaemia: results of two phase 1 studies. Lancet. 2020;396:1885-94.

66. Torikai H, et al. Toward eliminating HLA class i expression to generate universal cells from allogeneic donors. Blood. 2013;122:1341-9.

67. Kagoya Y, et al. Genetic ablation of HLA class I, class II, and the T-cell receptor enables allogeneic T cells to be used for adoptive T-cell therapy. Cancer Immunol Res. 2020;8:926-36.

68. Lee J et al. Abrogation of HLA surface expression using CRISPR/Cas9 genome editing: a step toward universal T cell therapy. Sci Rep 2020;10.

69. Gornalusse GG, et al. HLA-E-expressing pluripotent stem cells escape allogeneic responses and lysis by NK cells. Nat Biotechnol. 2017;35:765-72.

70. Taylor CJ, Peacock S, Chaudhry AN, Bradley JA, Bolton EM. Generating an iPSC bank for HLA-matched tissue transplantation based on known donor and recipient hla types. Cell Stem Cell. 2012;11:147-52.

71. Han J et al. CAR-engineered NK cells targeting wildtype EGFR and EGFRvIII enhance killing of glioblastoma and patient-derived glioblastoma stem cells. Sci Rep 2015;5.

72. Chu J, et al. CS1-specific chimeric antigen receptor (CAR)-engineered natural killer cells enhance in vitro and in vivo anti-tumor activity against human multiple myeloma. Leukemia. 2014;28:917-27.

73. Guven H, et al. Efficient gene transfer into primary human natural killer cells by retroviral transduction. Exp Hematol. 2005;33:1320-8.

74. Klingemann H. Challenges of cancer therapy with natural killer cells. Cytotherapy. 2015;17:245-9.

75. Tonn T, et al. Treatment of patients with advanced cancer with the natural killer cell line NK-92. Cytotherapy. 2013;15:1563-70.

76. Gong JH, Maki G, Klingemann HG. Characterization of a human cell line (NK-92) with phenotypical and functional characteristics of activated natural killer cells. Leukemia. 1994;8:652-8.

77. Müller T, et al. Expression of a CD20-specific chimeric antigen receptor enhances cytotoxic activity of NK cells and overcomes NK-resistance of lymphoma and leukemia cells. Cancer Immunol Immunother. 2008;57:41123. 
78. Mehta RS, Rezvani K. Chimeric antigen receptor expressing natural killer cells for the immunotherapy of cancer. Frontiers in Immunology. 2018;9:283.

79. Liu E, et al. Cord blood NK cells engineered to express IL-15 and a CD19-targeted CAR show long-term persistence and potent anti-tumor activity. Leukemia. 2018;32:520-31.

80. Liu E, et al. Use of CAR-transduced natural killer cells in CD19-positive lymphoid tumors. N Engl J Med. 2020;382:545-5.

A seminal paper showing feasibility in a phase I clinical trial of umbilical cord blood-derived CAR-NK cells in B cell malignancies.

81. Shah $\mathrm{N}$ et al. Antigen presenting cell-mediated expansion of human umbilical cord blood yields log-scale expansion of natural killer cells with anti-myeloma activity. PLoS One 2013;8.

82. Li Y, Hermanson DL, Moriarity BS, Kaufman DS. Human iPSC-derived natural killer cells engineered with chimeric antigen receptors enhance anti-tumor activity. Cell Stem Cell. 2018;23:181-92.e5.

83. Chang C, et al. FT819: translation of off-the-shelf TCRLess Trac-1XX CAR-T cells in support of first-of-kind phase I clinical trial. Blood. 2019;134:4434.

84. Exley $\mathrm{M}$, et al. CD1d structure and regulation on human thymocytes, peripheral blood T cells, B cells and monocytes. Immunology. 2000;100:37-47.

85. Nickoloff BJ, Wrone-Smith T, Bonish B, Porcelli SA. Response of murine and normal human skin to injection of allogeneic blood-derived psoriatic immunocytes: detection of $\mathrm{T}$ cells expressing receptors typically present on natural killer cells, including CD94, CD158, and CD161. Arch Dermatol. 1999; 135:546-52.

86. Chaidos A, et al. Graft invariant natural killer T-cell dose predicts risk of acute graft-versus-host disease in allogeneic hematopoietic stem cell transplantation. Blood. 2012;119:5030-6.

87. Leveson-Gower DB, et al. Low doses of natural killer T cells provide protection from acute graft-versus-host disease via an IL-4-dependent mechanism. Blood. 2011;117:3220-9.

88. Schneidawind D, et al. CD41 invariant natural killer T cells protect from murine GVHD lethality through expansion of donor CD41CD251FoxP31 regulatory T cells. Blood. 2014;124:3320-8.

89. Rotolo A, et al. Enhanced anti-lymphoma activity of CAR19-iNKT cells underpinned by dual CD19 and CD1d targeting. Cancer Cell. 2018;34:596-610.e11.

90. Kalyan S, Kabelitz D. Defining the nature of human $\gamma \delta$ T cells: a biographical sketch of the highly empathetic. Cellular and Molecular Immunology. 2013;10:21-9.
91. Kabelitz D, Wesch D, He W. Perspectives of $\gamma \delta \mathrm{T}$ cells in tumor immunology. Cancer Research. 2007;67:5-8.

92. Gentles AJ, et al. The prognostic landscape of genes and infiltrating immune cells across human cancers. Nat Med. 2015;21:938-45.

93. Silva-Santos B, Serre K, Norell H. $\gamma \delta$ T cells in cancer. Nature Reviews Immunology. 2015;15:683-91.

94. Cutmore LC, Marshall JF. Current perspectives on the use of off the shelf car-t/nk cells for the treatment of cancer. Cancers 2021;13.

95. Rozenbaum M, et al. Gamma-delta CAR-T cells show CAR-directed and independent activity against leukemia. Front Immunol. 2020;11:1347.

96. Neelapu SS, et al. First-in-human data of ALLO-501 and ALLO-647 in relapsed/refractory large B-cell or follicular lymphoma (R/R LBCL/FL): ALPHA study. $J$ Clin Oncol. 2020;38:800.

Preliminary data for the feasibility and activity of the first allogeneic CAR-T product in lymphoma.

97. First allogeneic CAR T-cell therapy impresses in relapsed/refractory lymphoma. Oncologist 2020;25:S4.

98. First-in-human data of ALLO-501A, an allogeneic chimeric antigen receptor (CAR) T-cell therapy and ALLO647 in relapsed/refractory large B-cell lymphoma $(\mathrm{R} / \mathrm{R}$ LBCL): ALPHA2 study. J Clin Oncol. https://doi.org/ 10.1200/JCO.2021.39.15_suppl.2529.

99. Preliminary safety and efficacy of PBCAR0191, an allogeneic, off-the-shelf CD19-targeting CAR-T product, in relapsed/refractory (r/r) CD19+ NHL. J Clin Oncol. https://doi.org/10.1200/JCO.2021.39.15_suppl.7516.

100. A phase 1 dose escalation and cohort expansion study of the safety and efficacy of allogeneic CRISPR-Cas9engineered T cells (CTX110) in patients (Pts) with relapsed or refractory $(\mathrm{R} / \mathrm{R}) \mathrm{B}$-cell malignancies (CARBON). J Clin Oncol. https://doi.org/10.1200/ JCO.2021.39.15_suppl.TPS7570.

101. Chavez JC, et al. Updated outcomes with axicabtagene ciloleucel (axi-cel) retreatment (reTx) in patients (pts) with relapsed/refractory (R/R) indolent non-Hodgkin lymphoma (iNHL) in ZUMA-5. J Clin Oncol. 2021;39:7548.

\section{Publisher's note}

Springer Nature remains neutral with regard to jurisdictional claims in published maps and institutional affiliations. 